\title{
Effects of Light's Colour Temperatures on Visual Comfort Level, Task Performances, and Alertness among Students
}

\author{
B.M.T Shamsul, C.C. Sia, Y.G Ng, K. Karmegan ${ }^{*}$ \\ Department of Environmental and Occupational Health, Universiti Putra Malaysia, Selangor, Malay sia \\ *Corresponding author: uvea_2000@y ahoo.com \\ Received December 31, 2012; Revised July 18, 2013; Accepted July 19, 2013
}

\begin{abstract}
Introduction: Correlated colour temperatures (CCT) of the light source in indoor environment plays an imperative role in addressing both psychological and physiological functions of the occupant. As one of the determinants of lighting quality, CCT are off particular importance which affects quality of work and in classroom learning. Objective: The aim of this study is to determine the effects of warm white light (WWL) (CCT = 3,000K), cool white light $(\mathrm{CWL})(\mathrm{CCT}=4,000 \mathrm{~K})$ and artificial daylight $(\mathrm{DL})(\mathrm{CCT}=6,500 \mathrm{~K})$ on the performances, subjective alertness level, visual comfort level and preferences of student in Faculty of Medicine and Health Sciences, Universiti Putra Malaysia. Methodology: A laboratory controlled experiment was conducted on total of 47 undergraduate students volunteered to participate in a series of test under three coloured light sources. FrACT software was used to assess visual task performance, modified OLS questionnaire was used to evaluate subjective comfort level and preferences, typing test and KSS alertness level monitoring was conducted. Result: Significant increase was observed in subjective alertness level $(p=0.041)$ and computer-based performances $(p=0.001)$ under DL condition in relative to WWL condition. In terms of typing performances, respondents performed significantly better in term of typing speed under CWL than DL and WWL. Least typing errors were made under DL, followed by CWL and WWL. CWL is the most preferred $(p=0.001)$ and most comfortable $(p=0.011)$ CCT environment where subjects indicated the ability to perform task longer in this coloured-lit environment. Conclusion: The study concludes that the CWL and DL were more beneficial for alertness level and academically activities for both computer-based and paper-based activities.
\end{abstract}

Keywords: lighting, correlated colour temperatures, visual comfort, alertness level, visual task performance

Cite This Article: Shamsul, B.M.T, C.C. Sia, Y.G Ng, and K. Karmegan, "Effects of Light's Colour Temperatures on Visual Comfort Level, Task Performances, and Alertness among Students.” American Journal of Public Health Research 1, no. 7 (2013): 159-165. doi: 10.12691/ajphr-1-7-3.

\section{Introduction}

Lighting quality is one of the determinants of indoor environment quality. Various study has been conducted to compare the effects of different lighting conditions on health, productivity, well-being and alertness level (van Bommel and van den Beld, 2004; Hameed and Amjab, 2009; Boyce, Veitch, Newsham, Myer and Hunter 2003; Altomonte, n.d).

With regards to human perception, correlated colour temperature (CCT) and illu minance level are the two most important characteristics of light to be considered (Veitch and Nesham, 1998; Barkman, Wessolowski and SchulteMarkwort, 2011; Sanaz, 2011). CCT is an essential factor in the learning place because it supports and enhances the impact of lighting on users (Sanaz, 2011).

The application of correct CCT environment can be beneficial to occupants. On the other hand, the use of inappropriate CCT of light has significant effects on human health, such as eyes strain, or effects to the emotion and human circadian system (van Bommel and van den Beld, 2004; Mills, Tomkins and Schlangen, 2007; Halonen, Tetri and Bhusal, 2010) which further cause loss of productivities.

The aim of this study was to compare the differences of students' performances and well-being when exposed to light of CCT with $3,000 \mathrm{~K}, 4,000 \mathrm{~K}$ and $6,500 \mathrm{~K}$. In particular alertness, objective visual performance, typing speed and accuracy as well as subjective visual comfort assessment were tested in order to determine optimum CCT environment for students in Faculty of Medicine and Health Sciences, Universiti Putra Malaysia.

\section{Methodology}

\subsection{Subject}

This is a repeated-measures experimental study design involving 47 young and healthy undergraduate students (19 males, 28 females), from the Faculty of Medicine and Health Sciences (FMHS), Universiti Putra Malaysia 
(UPM). Purposive sampling was applied to select only students with normal or corrected visual acuity.

The mean average age of respondent recruited for the study was $21.49 \pm 1.3$ years. The experiments was conducted during daytime from 29th February 2012 until 18th April 2012. It was scheduled in such a way that each subject performed the test only once on each day.

\subsection{Setup of Experiment}

The experiment was conducted in a confined room (with no window - external source of light) inside the Occupational Health and Safety Laboratory, FMHS, UPM. The dimension of the room was $6.2 \mathrm{~m} \times 3.1 \mathrm{~m} \times 2.8 \mathrm{~m}$ where the room was being illuminated by fluorescence light tubes installed to the housing in the ceiling.

For the WWL $(3,000 \mathrm{~K})$ environment, the room was illuminated by Philips' MASTER (PL-L 36W/830/4P) light tube. On the other hand, CW L $(4,000 \mathrm{~K})$ environ ment uses light tubes of Tian He's Tricolour (36W) whereas DL (6,500K) environ ment was created by using Liyoda's light tube (PLL*36W/EX-D). The luminance level was controlled in all three environment (Figure 1).
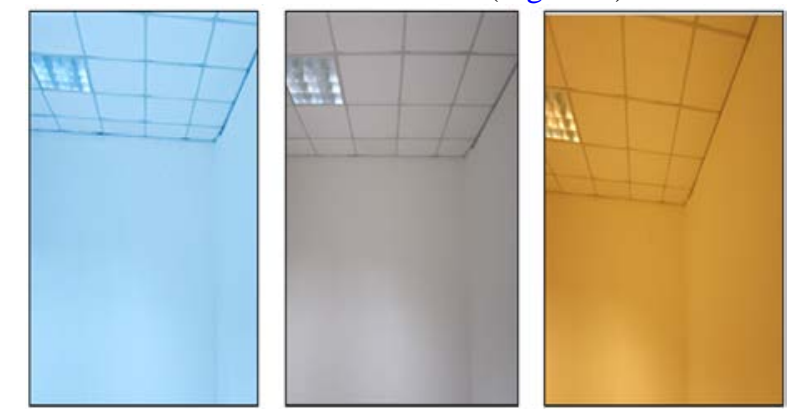

Figure 1. Photographs of the three different CCT of light, taken with digital camera. From left to right: $3,000 \mathrm{~K}, 4,000 \mathrm{~K}$, and 6,500K light environment (CCT)

\subsection{Instruments}

\subsubsection{Alertness}

Changes of alertness in each of the respondent was determined using Karolinska Sleepiness Scale (KSS) which has been validated against EEG data by A kerstedt and Gillberg (1990). It is a subjective rating where each respondent state their current alertness level on a 9-stage scale beginning from "extremely alert" (1) to "very sleepy”, "great effort to keep awake” and "fighting sleep” (9).

\subsubsection{Performance at Computer-based Task}

To compare the influence of the three different CCT; WWL, CWL and DL on the performance of the respondents during computer-based task, the "Freiburg Visual Acuity and Contrast Test” (FrACT) was being used to determine 1) visual acuity and 2) contrasting threshold. The methods and procedures benchmarked and modified by Linhart's study (2011) was used - correct recognition of Landolt rings on a PC screen.

The complete FrACT test is as illustrated in Figure 2. Each part present different tests where (a) visualizes the use of the four direction keys as a guide while (b) present examples of the acuity task and (c) the contrast task.

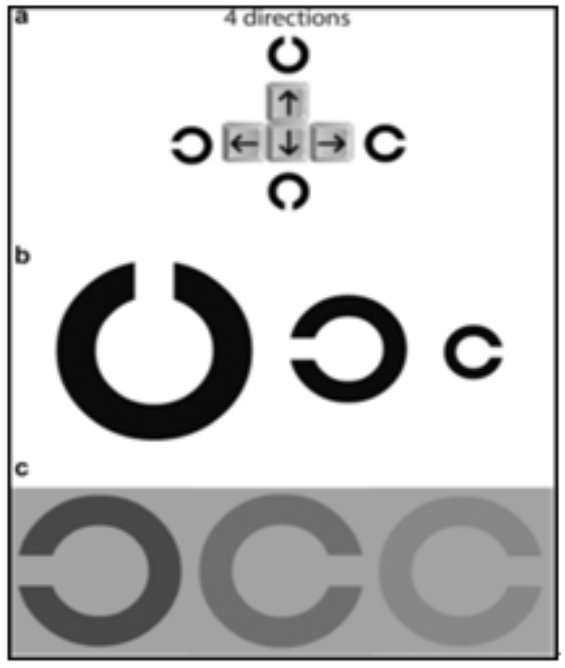

Figure 2. Overview of the computer-based FrACT test

In the test of visual acuity (b), a sequence of three Landolt rings of different sizes and orientations were presented on the computer screen. The respondents determine the orientation of the ring and give corresponding answer by clicking on external keyboard as quickly as possible.

For each sequence, the performance indicators were determined as follow calculation: (Number of correct responses / Total duration of sequence).

As for contrast threshold task, the sizes of the Landolt rings were maintained but the contrast between rings and the screen background was modified during the test. Then, the contrast threshold was determined by the software itself for every sequence. Average of the contrast threshold for all three trials would be taken as indicator.

For the purpose to test the influence of the three different CCT of light on the subjects' performance during a paper-based task, we had again referred to tests method used in a study by Linhart and Scartezzini (2011).

\subsubsection{Perfor mance at Paper-based Task}

Similar to the computer-based task, Landolt rings were used for the paper-based task. Each respondents received a piece of white paper on which rows of Landolt rings are printed (very weak contrast) as shown in Figure 3. They were asked to determine, within 5 minutes and without writing on the paper, the correct orientations of all the 96 rings by writing down the number of counted rings for all four possible orientations (open on top, bottom, left, or right). Then, the numbers of mistakes were counted as the performance for paper-based task for each different light environment.

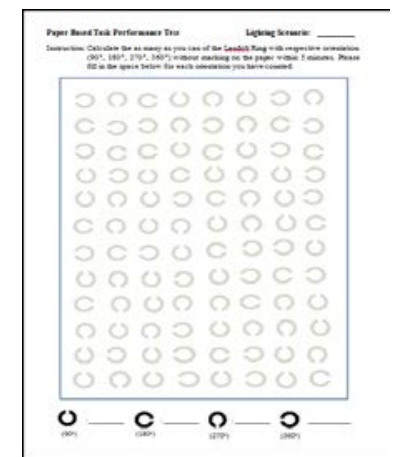

Figure 3. Overview of paper-based FrACT test. 


\subsubsection{Visual Comfort and Subjective Preferences Ass essment}

A modified Office Lighting Survey (OLS) questionnaire was used to determine the satisfaction of respondent during each light environment. A mix of general and artificial lighting-specific statements were asked using the questionnaire. Respondent were asked to rate their agreement with each statement on a 4-responses (yes, rather yes, rather no, no) answering scale with no neutral choice. The questions asked and scoring in OLS were as detailed in Table 1 as follow:

Table 1.Questions and scoring for positive and negative statement asked in OLS

\begin{tabular}{|c|c|c|}
\hline \multicolumn{2}{|r|}{ Questions } & Score \\
\hline \multirow{6}{*}{ 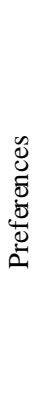 } & 1. like the light ing in this office. & \multirow{4}{*}{$\begin{array}{c}\text { Yes }=+3 \\
\text { Rather yes }=+2 \\
\text { Rather no }=+1 \\
\text { No }=0\end{array}$} \\
\hline & $\begin{array}{l}\text { 2. In general, the lighting in this office is } \\
\text { comfortable. }\end{array}$ & \\
\hline & $\begin{array}{l}\text { 3. This colour of light allows me to carry } \\
\text { out the different tasks. }\end{array}$ & \\
\hline & 4. My skin looks natural under the light. & \\
\hline & 5. The lighting in this office is too warm. & \multirow{8}{*}{$\begin{array}{c}\text { Yes }=+3 \\
\text { Rather yes }=+2 \\
\text { Rather no }=+1 \\
\text { No }=0\end{array}$} \\
\hline & 6. The lighting in this office is too cold. & \\
\hline \multirow{6}{*}{ 离 } & 7. I feel eye strain. & \\
\hline & 8. My eye lids are heavy. & \\
\hline & 9. My eyes feel dry. & \\
\hline & 10. I have burning eyes. & \\
\hline & $\begin{array}{l}\text { 11. I have a headache working under this } \\
\text { CCT of light. }\end{array}$ & \\
\hline & $\begin{array}{l}\text { 12. I have difficulties in seeing objects on } \\
\text { the screen. }\end{array}$ & \\
\hline
\end{tabular}

Preferences of respondents were assessed using Question 1 to 6, while visual comfort level was determined from Question 7-12. For every response made for Question 1 to 4 (positive statements), scores are given for each questions where +3 points were given if 'Yes' as the selected response, +2 for 'Rather Yes', +1 for 'Rather No' and 0 for 'No'.

For Question 5 to 12 (negative statements), +3 points were given for 'No', +2 for 'Rather No', +1 for 'Rather yes' and +0 for 'Yes'. Standard scores were generated for rating of preferences and visual co mfort level, respectively.

\subsubsection{Typing Performance Test}

To determine the optimum CCT environ ment for typing performance, subjects were given 10 minutes to type an article referring to printed article. As shown in Figure 4, three different articles (approximately 400 words) were prepared and clipped on a document holder for respective environment (CCT of light).

Using Microsoft Office Word 2007 as the word processor software, the automatic spelling and grammar checking were disabled prior to test. The typing speed (total numbers of words typed) and typing accuracy (percentage of typing errors through document analysis) of the respondents for every environment (CCT of light) were then recorded.

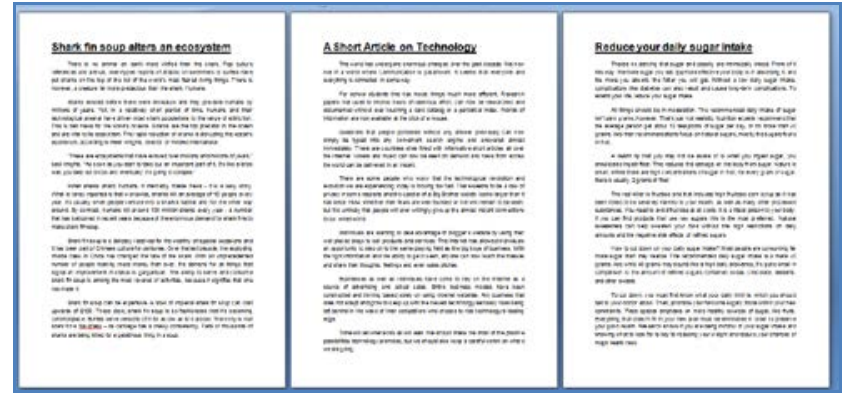

Figure 4. Different printed articles for participants to replicate in typing performance test

\subsubsection{Statistical Analysis}

Three types of statistical analysis tests were used in this study. Descriptive statistics (percentage, mean and standard deviation) were used to describe the sociodemographic distribution of the population. Comparison of visual comfort level, subjective preferences, visual task performances, and typing performance were performed with repeated-measures ANOVA test. Alertness level before and after experiment was analyzed using pairedsample T-test. Statistical Package for Social Sciences (SPSS), version 19 was used in all analysis. Statistical significance ( $\mathrm{p}$-value) is determined at $\mathrm{p}<0.05$.

\subsection{Study Procedures}

The study procedures consist of two phase; intake and the experiment phase. During the intake phase, respondents were informed about the study and the experimental procedures. A written permission form was then given to respondents who decided to participate in the study to be signed and returned before the experimental proceeds.

For the experiment phase, we had randomized the sequence of CCT of light. Each trial was completed in an average of 50 minutes. The sequence of the study was shown in Figure 5. In the beginning, the respondent were allowed to adapt to the current lighting environment for 15 minutes. When the respondent were ready, the test began.

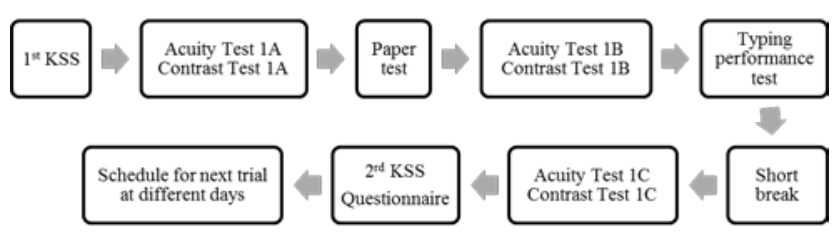

Figure 5. Flow of procedures of our experiment

Respondents had to fill out a KSS-test before performing a computer-based acuity-test and a computerbased contrast-test (to determine the orientation of Landolt rings in both cases). After that, the paper-based task was carried out. The latter was then followed by another sequence of acuity- and contrast-tests, and typing test for about 10 minutes.

Subsequently, respondents were given a short break to rest. During the break, they were offered to watch hilarious video or simply chatting. Respondent then took a third sequence of acuity- and contrast-tests and second KSS-test. The experiment was completed by filling out the modified OLS questionnaire. Lastly, appointment for next environment (different CCT of light) was made and the respondent was allowed to leave. 
The results of the test (e.g. correct answers, response time per ring) were recorded and stored in a Microsoft Office Excel 2007 spreadsheet. The sequence of the experiment were repeated for the rest of the CCT of light.

\section{Result}

\subsection{Subjective Alertness}

Figure 6 shows the comparison bar chart on effects of different light source (CCT) on respondents' subjective alertness level. Comparing pre-experiment alertness level to the post-experiment alertness level under respective light source, significant increase of alertness was observed for DL ( $<<0.05)$.

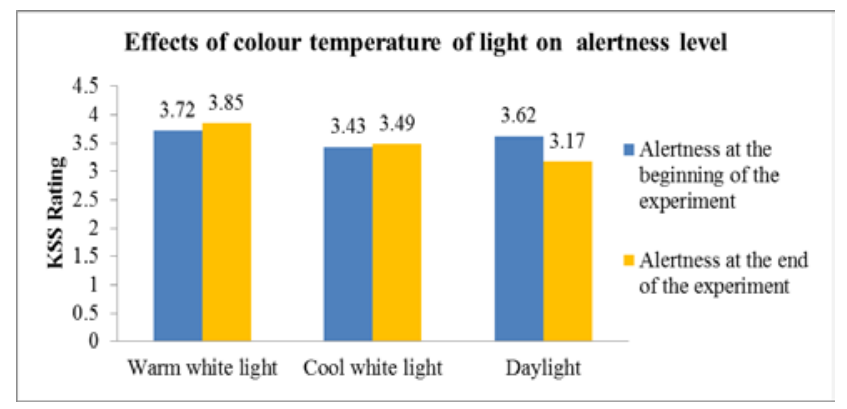

Figure 6. Comparison of alertness level before and after the trial between light sources

However, although the raw mean result of the alertness level under WWL and CWL decreases in post-experiment from pre-experiment, the observed differences of alertness level in WWL environment and CWL environment were not significantly different $(\mathrm{p}>0.05)$ respectively.

In our further analysis comparing alertness level across 3 different environment (CCT of light), the preexperimental alertness level was not statistically different. However, when post-experiment alertness level was compared, average KSS rating of respondents in DL environment was found to be significantly lower (more alert) than WWL environment $[F(2,92)=4.121$; p < 0.05].

\subsection{Performance at Computer-based Task}

Figure 7(a) gives an overview of the results obtained during the computer-based tasks performed under three different source of light (CCT). In our analysis significant differences was found between these three environment (CCT of light) ( $\mathrm{p}$ 0.05). In comparis on, we found that the performance value under DL was significant better than WWL $(p<0.001)$ with the mean difference of 0.079 correct decisions per second.

In terms of contrast threshold perception, the average performance in contrast threshold under the three different source of light (CCT) was within $0.61 \%$ to $0.69 \%$. Although the observed raw mean score (Figure $7 \mathrm{~b}$ ) shows differences, no statistical significant difference $(p>0.05)$ were found between them.

\subsection{Performance at Paper-based Task}

For performance on paper-based task, the average number of incorrectly identified ring's orientation under each environment (CCT of light) was compared to one another. The respondents scored the best (mean of 2.47 errors) under WWL, followed by CWL and DL (mean of 2.70 errors respectively). Nevertheless, the differences between these values were not statistically significant ( $p>$ 0.05).

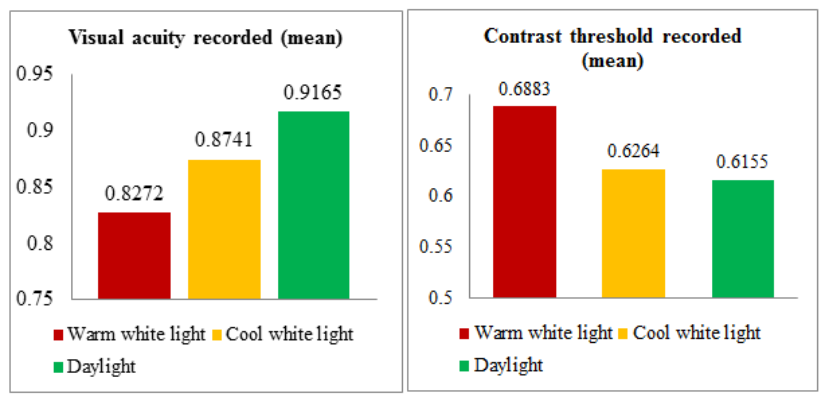

Figure 7. (a): Average results of the acuity test (correct decision per second); and (b): contrast threshold (\%) obtained during the computerbased tasks

\subsection{Subjective Preferences and Visual Comfort}

In terms of preferences, CWL (14.638 points) was most preferred by the respondents, in relative to WWL (12.085 points) and DL (13.702 points) as shown in Figure 8. There were statistically significant difference among the three source of light (CCT) [F $(2,92)=7.303$, p < 0.05] where CWL was significantly $(\mathrm{p}<0.0001)$ better preferred to WWL with mean difference of 2.55 .

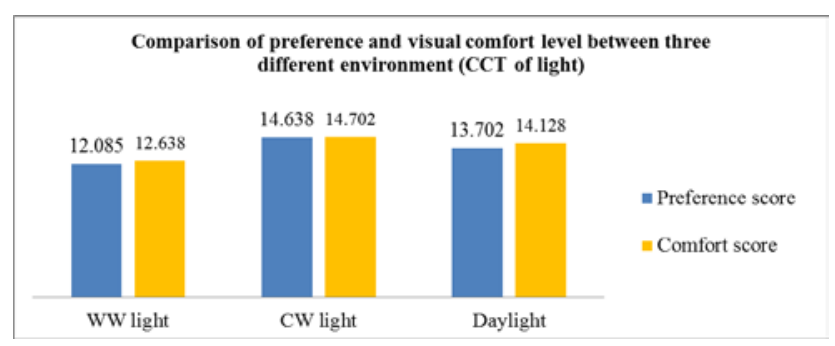

Figure 8. Comparison of subjective preference and comfort level of participants for three different CCT of light

Where visual comfort is concerned, CWL was correspondingly perceived as the most comfortable light's CCT (14.702 points) compared to WWL (12.638 points) and DL (14.128 points).

Similarly, statistical analysis yield significant differences $[F(2,92)=4.739 ; p<0.05]$ among the three environment (CCT of light) where visual comfort level of respondent working under CWL reported significantly ( $p$ $<0.005$ ) higher comfort with a mean difference of 2.064 points as compared to WWL.

\subsection{Typing Performance Test}

Table 2 shows the results of the typing performances, including typing speed and typing accuracy of respondents under three different environments (CCT of light). Based on the raw mean score, the most word (faster speed) is typed in CWL environment but DL environment was better for typing accuracy.

Analysis using repeated-measures ANOVA shows that the typing speed among the three environment was significantly different $[\mathrm{F}(2,92)=10.829$; $\mathrm{p}<0.05]$. In 
our further analysis, pairwise comparison of typing speed revealed that respondents could type in significantly faster under CWL ( $p<0.0001$ ) followed by DL ( $p=0.010<$ 0.05 ) as compared to WWL. The mean differences were 11.70 words and 19.53 words respectively.

Table 2. Comparison of typing performances of subjects in three different lighting conditions

\begin{tabular}{ccc}
\hline CCT & $\begin{array}{c}\text { Typing speed } \\
\text { (Mean } \pm \text { S.D.) }\end{array}$ & $\begin{array}{c}\text { Typing } \\
\text { accuracy } \\
\text { (\% of error) }\end{array}$ \\
\hline $\begin{array}{c}\text { Warm White Light, } \\
\text { WWL (3,000K) }\end{array}$ & $\begin{array}{c}263.36 \pm 71.93 \\
\text { words }\end{array}$ & $3.36 \pm 2.45$ \\
CoolWhite Light, \\
CWL (4,000K) & $\begin{array}{c}282.89 \pm 71.68 \\
\text { words }\end{array}$ & $2.47 \pm 2.04$ \\
Daylight, DL (6,500K) & $\begin{array}{c}275.06 \pm 66.93 \\
\text { words }\end{array}$ & $2.25 \pm 1.87$ \\
\hline
\end{tabular}

As for typing accuracy, statistical analysis shows that there was significant differences $[F(1.639,75.376)=$ 10.829, $\mathrm{p}<0.05$ ] between these three environment (CCT of light) - Greenhouse-Geisser correction was made as Mauchly's Test of Sphericity ind icated that the assumption of sphericity in typing speed has been violated $\left[\chi^{2}(2)=\right.$ 11.212; $\mathrm{p}<0.05]$.

Further analysis indicate that the respondents could perform in significantly better $(p<0.0001)$ accuracy under DL as compared to $\mathrm{WW}$ light where the mean difference was $1.11 \%$ of typing error.

\section{Discussion}

These results show that the three tested CCT of light sources are comparable in terms of visual comfort level, subjective preference, task performances, and alertness level.

\subsection{Subjective Alertness}

Figure 6 shows that the subjective alertness level was averagely situated between "rather alert" and "alert" under all three different source of light (CCT). Increased alertness level under DL has been observed in many previous studies (Rautkylä, Halonen, and Lehtovaara, 2008; Chellappa, et al, 2011; Halonen, et al, 2010).

They found that the higher CCT of light during lecture led to higher alertness level of the students at the end of the study. This was probably associated to improved mental activity level and autonomic nervous system of human triggered by higher CCT of light.

This was corresponding to findings in an intervention study (Viola, James, Schanglen and Djik, 2008) comparing the use of white light $(4,000 \mathrm{~K})$ and blueenriched white light $(17,000 \mathrm{~K})$ among office workers where significant improved alertness was observed with various other findings including reduction of daytime sleepiness.

\subsection{Performance at Computer-based Task}

As displayed in Figure 7(a), computer-based task under DL led to best performance in acuity test among respondents. The finding was similar to study by Berman, Fein, Jewett and Ashford (1994) who discovered that the whiter light (higher CCT) induced a smaller pupil size, and this created better visual performance in looking at small size objects.

From the trend of result for acuity test, it can be inferred that the CCT is proportional with the computer task performance. This was suggested by a study by Juslen and Tenner (2005) that the productivity human increased when the CCT increased for $5.7 \%$.

In Figure 7(b), respondents had lowest contrast threshold (highest sensitivity) during DL light condition. Nevertheless, as there were no significant differences observed, there was a considerable probability that the variations observed of the raw mean score may have occurred by chance.

Similarly to the study done by Michiko, Hatsunori and Hiroyasu (2001), a comparis on of different CCT $(3,000 \mathrm{~K}$ vs. 5,000K vs. 6,700K) found no systematic difference on contrast threshold. However, the lack of finding was suggested by Linhart and Scartezzini, (2011) that this may indicate that the FrACT test used during our study was inappropriate; for example, it might have not be sensitive enough to test respondents' contrast threshold.

\subsection{Performance at Paper-based Task}

For the of paper-based task performance, it was not significantly different across different CCT. Least errors were made in WWL environment was observed. Although van Bommel and van den Beld (2004) propose that WWL is related to relaxing emotion and co zy atmosphere, which might facilitated respondents in this task, no major studies has supported such suggestion. Thus the results rather reflect that the differences between the three environment (CCT of light) were not strong enough to have measurable effects on this dependent variable.

\subsection{Visual Comfort and Subjective Preferences Assessment}

Figure 8 shows overall trend for subjective preference and visual comfort level for each CCT of light tested among the respondents. The preferences of CCT of light were strictly subjective as the same person may prefer different CCT at various times during the day (Miller, 2007; van Bommel and van den Beld, 2004). In this study, CWL met respondents' expectation in these two parameters.

In a recent study, Park, Chang, Kim, Jeong and Choi (2010) found that $55 \%$ of males and $42 \%$ of females preferred $4000 \mathrm{~K}$ condition compared to $3,000 \mathrm{~K}, 5,000 \mathrm{~K}$ and $6,000 \mathrm{~K}$ in an office survey for CCT preference on personal CCT. However, different researcher found different personal preference as Miller (2007) stated that at daylight levels (500 lux), the average preferred colour temperature was around 3,300K (Miller, 2007) while Sanaz (2011) indicated that people prefer warmer CCT and dim light.

In terms of visual comfort, respondents reported significantly better comfort under CWL as compared to WWL. The reasons of visual discomfort under WWL were supported in OLS where $31.9 \%$ of subjects had experienced mild eyes strain and $21.3 \%$ had dry eyes during the experiment.

The finding was in parallel with Manaz (2007), where $4,000 \mathrm{~K}$ was preferred to $2,700 \mathrm{~K}$ in terms of visual comfort and spaciousness in office. However, few studies 
found contradicting conclusion where $3,000 \mathrm{~K}$ light source was comfortable and high CCT $(17,000 \mathrm{~K})$ lead to dizziness (Park et al., 2010; Górnicka, 2008).

Taken into consideration of different cultural and regional factors, the finding of preferences and comfort level in this study was perhaps relevant and applicable to undergraduate students in this faculty.

\subsection{Typing Performance Test}

Table 2 shows that average typing speed of respondents was statistically fastest under CWL. We believe that the speed of typing were associated with visual comfort although the experiment was not set up to test this. Our postulation was that, as respondents felt more comfortable under CWL, they typed significantly faster than WWL (least comfortable perceived). The finding was supported by finding from another research by Park et al. (2010), where their work performance under CWL condition was shown to be higher than WWL.

For typing accuracy, DL was observed to result in least typing errors. We attributed the significant better performance to be related to increased alertness level under high CCT as supported by a study (Lehrl, Gerstmeyer, Jacob, Frieling, Henkel, Meyrer, Wiltfang, Kornhuber and Bleich, 2007) which pointed out that WWL was less effective in information processing (reading material on paper) and implementation (typing).

\section{Conclusion and Recommendation}

Overall, the respondents preferred the CWL as compared to other CCT of light, and they agreed that CWL was visually the most comfortable light sources. Respondents reported that they could imagine themselves working comfortably under this light source for more than 6 hours, which was longer than in WW light and DL condition.

Nevertheless, it was observed that their subjective alertness level and performance in computer-based acuity task including typing accuracy was the best under DL although CWL was significantly better for typing speed. There were however no significant differences in the computer-based contrast tasks and paper-based test.

This led us to the conclusion that there is no absolute 'perfect light (CCT)', although in our study, CWL and DL were found to be more beneficial to undergraduate students than the WWL, which is the default lighting in used in FMHS, UPM.

Thus, we recommend to the technical management of academic institution on the preference and reinstallation of new lighting system (CCT) in the near future for the sake of better performance of undergraduate students. In addition, we suggest that different occupants to be given different option for higher CCT of light when performing activities or tasks requiring high attention (e.g. microscopic works and solving complex equation), while moderate CCT is suitable for activities that need longer duration, such as typing.

Lastly, the results of this study are to be recommended to architect, engineers or lighting designers as a reference to be considered in order to achieve better indoor environment quality.
Further work could look at the long-term effects of different CCT of light on performance and visual comfort of students, or on office workers. Also, more study on actual environment is warranted before further conclusion can be drawn about human's sensitivity on effects of lighting with high and low CCT. It would be more favorable to include new tests that have clear and valid scoring method, especially in measuring human's nonvisual effects.

\section{Limitations of the Study}

Some limitations of the study should be acknowledged. This study had included respondents from only one faculty, which makes generalizing the findings somewhat difficult. Also, the study included self-reported perceptions, which might be biased in terms of the reliability of measurements.

Besides that, as in other experimental design on effects of lighting of the study in the past, it is almost impossible to implement a double-blinded design. Therefore, it is unavoidable for the results to be at risk of influence of Hawthorne effect, where respondents might perform outstandingly well (sometimes bad), as they know that they were being observed and cared.

\section{Acknowledgement}

The author is grateful to Associate Professor Dr. Shamsul Bahri Tamrin (UPM) for his meticulous care and motivating advice throughout the research progression. Also, appreciation goes to the Department of Environmental and Occupational Health, Faculty of Medicine and Health Sciences, UPM, for their support in the development and design of this study.

\section{References}

[1] Åkersted, T., and Gillberg, M., "Subjective and objective sleepiness in the active individual,". International Journal of Neuroscience, 52(12).29-37.1990.

[2] Altomonte, S. (n.d.), "Lighting and Physiology: Artificial and natural lighting and its relation to human body," [Online]. Available: http:// www.melbourne.vic.gov.au/.../Study3TechnicalPaper_updated.D OC. [Accessed Oct. 15.2011].

[3] Barkmann, C., Wessolowski, N., and Schulte-Markwort, M, "Applicability and efficacy of variable light in schools," Physiology and Behavior, Sept. 2011. doi:10.1016/j.physbeh.2011.09.02.

[4] Berman, S.M., Fein, G., Jewett, D.L., and Ashford, F, "Landolt C recognition in elderly subjects is affected by scotopic intensity of surround illuminants," Joumal of Illuminating Engineering Society 199, 23(2).1994.

[5] Boyce, P. R., Veitch, J. A., Newsham, G. R., Myer, M. and Hunter, C, "Lighting quality and office work: A field simulation study (PNNL-14506)," 2003. [Online]. Avaiable: http: //www.nrccnrc.gc.ca/obj/irc/doc/pubs/b3214.1/B3214.1.pdf. [Accessed Oct.22.2011].

[6] Chellappa, S. L., Steiner, R., Blattner, P., Oelhafen, P., Götz, T., and Cajochen, C, "Non-visual effects of light on melatonin, alertness and cognitive performance: Can blue-enriched light keep us alert,” 2011. [Online]. Available:

http: //www.virtualdaylight.com/downloads/ArtDayErgoLight.pdf. [Accessed April. 18.2012]. 
[7] Górnicka, G, Lighting at work: Environmental study of direct effects of lighting level and spectrum on psychophysiological variables, Technische Universiteit, Eindhoven, 2008.

[8] Halonen, L., Tetri, E., and Bhusal, P, Guidebook on energy efficient electric lighting for buildings: Chapter 3: Lighting Quality, Aalto University School of Science and Technology, Finland, 2010, 41-56.

[9] Hameed, A. and Amjab, S, "Impact of Office Design on Employees' Productivity: A Case study of Banking Organizations of Abbottabad Pakistan,” Journal of Public Affairs, Administration and Management, 3(1).2009.

[10] Juslen, H. and Tenner, A. "Mechanisms involved in the enhancing human performance by changing the lighting in the industrial workplace” Intemational Joumal of Industrial Ergonomics, 3. 843855. 2005.

[11] Lehrl, S., Gerstmeyer, K., Jacob, J.H., Frieling, H., Henkel, A.W., Meyrer, R., Wiltfang, J., Kornhuber, J., and Bleich, S,“Blue light improves cognitive performance,” Journal Neural Transmission. 114. 457-460.2007.

[12] Linhart, F. and Scartezzini, J. L, "Evening office lighting - visual comfort vs. energy efficiency vs. performance," Building and Environment, 46, 981-989. 2011.

[13] Manav, B, “An experimental study on the appraisal of the visual environment at offices in relation to colour temperature and illuminance,” Building Environment 42, 979-983. 2007.

[14] Michiko, I., Katsunori, O. and Hiroyasu, U, "Change in Visual Contrast Sensitivity with Age Depends on Illuminance Level," Journal of the Illuminating Engineering Institute of Japan, 85(5), 352-360. 2001.

[15] Miller, H. Bright Idea: Personal Control for Office Lighting. Research Summary. 2007. [Online]. Available: http://www.hermanmiller.com/research/researchsummaries/bright-idea-personal-control-for-office-lighting.html. [Accessed July, 2011].
[16] Mills, P. R., Tomkins, S. C., \& Schlangen, L. J. (2007). The effect of high correlated colour temperature office lighting on employee wellbeing and work performance. Journal of Circadian Rhythms, $5(1), 2$.

[17] Noguchi, H., and Sakaguchi, T, "Effect of illuminance and color temperature on lowering of physiological activity. Joumal of Applied Human Science," 18.117-123. 1999.

[18] Park, B. C., Chang, J. H., Kim, Y. S., Jeong, J. W. and Choi, A. S, "A Study on the Subjective Response for Corrected Colour Temperature Conditions in a Specific Space," Indoor Building Environment, 19(6), 623-637.2010.

[19] Rautkylä, E., Halonen, L., Lehtovaara, J. (2008). Effects of artificial light spectrum on alertness: Vava - field study. Report 54, Light ing unit, Helsinki University of T echnology.

[20] Sanaz, A.S, “The Influence of Light on Student's Learning Performance in Learning Environments: A Knowledge Intemalization Perspective," Joumal of World Academy of Science, Engineering and T echnology, 81. 2011.

[21] Stephenson, P, “Artificial daylight as ergonomic lighting,” 2005. [Online]. Available: http://www.virtualdaylight.com/downloads/ArtDayErgoLight.pdf. [Accessed Sept.22, 2011].

[22] Van Bommel, W. J. M. and van den Beld, G. J, "Lighting for work: a review of visual and biological effects," Lighting Res. Technology, 36(4), 255-269.2004

[23] Veitch, J. A. and Newsham, G. R, "Deteminants of lighting quality 1: State of science," Joumal of the Illuminating Engineering Society 27(1), 92-106. 1998.

[24] Viola, A. U., James, L. M., Schlangen, L. J., \& Dijk, D. J. (2008). Blue-enriched white light in the workplace improves self-reported alertness, performance and sleep quality. Scandinavian Joumal of Work, Environment \& Health, 297-306. 